\title{
Management of pregnancy in women with congenital heart disease
}

The number of women with congenital heart disease reaching childbearing age is increasing, and this group now represents the majority of women with heart disease during pregnancy. This increase raises certain issues which have already been addressed in editorials in this journal, ${ }^{12}$ relating to risk factors and the difficulties arising from pregnant women with severe congenital heart disease.

\section{Simple defects}

Atrial and ventricular septal defects, and arterial duct, when operated on early in infancy, do not require any special treatment. Asymptomatic left-to-right shunts and moderate pulmonary stenosis are also usually well tolerated. These women should be able to give birth in non-specialist centres. The only concern is prevention of endocarditis in case of residual shunts or valvar regurgitation. Atrial septal defect is the most common congenital heart disease in adults, and in the absence of pulmonary hypertension induces barely any complications. ${ }^{3}$

\section{Contraindications}

Eisenmenger reaction, major or significant aortic dilatation in Marfan syndrome, severe aortic stenosis, deep cyanosis, and heart failure put the patient at high risk of maternal death, even where there is close follow up . Eisenmenger reaction carries up to $50 \%$ mortality. ${ }^{1}$ Recent inquiries in the $\mathrm{UK}^{4}$ report maternal death in the range of $2-11$ / 100000 from 1994-96. The main cardiac causes of death are pulmonary hypertension or dissecting aneurysm.

Recommendations for Eisenmenger patients include avoidance of vasodilatation, maintenance of preload, and use of prophylactic heparin. ${ }^{1}$ Marfan disease with aortic dilatation should contraindicate pregnancy when the ascending aorta is more than $40 \mathrm{~mm}$ as there is an increasing risk of aortic dissection. ${ }^{5}$ Even if the aorta is smaller, pregnancy should be followed up closely with monthly echocardiograms.

\section{Palliated or balanced heart defect}

Difficult issues can also arise in women who have undergone palliative surgical procedures. These patients lives are not usually at risk but they may suffer morbidity and the fetus may be harmed.

Understanding the physiological changes induced by pregnancy can help to anticipate or prevent complications: an increase in blood volume $(40 \%)$, a fall in peripheral resistance, an increase in cardiac output (40\%), and an altered heart rate are the most important of these changes. Potential for thrombosis is high during pregnancy and prolonged several weeks after delivery. Labour and delivery cause the blood volume and systemic resistance to vary greatly.

Pregnant women with congenital heart disease can be divided into five groups:

(1) The systemic ventricle is the right ventricle, as in transposition of the great arteries having undergone an atrial switch, or in double discordance. Adding to potential arrhythmias induced by surgery, the risk is deterioration of the right ventricular function with increasing atrioventricular regurgitation, sometimes irreversible after pregnancy. Several studies have reported good outcomes of pregnancy in selected women with atrial switch and with double discordance. ${ }^{367}$

(2) Overloaded right ventricle caused by pulmonary regurgitation and/or obstruction. This is often the case in the following situations: operated tetralogy of Fallot, right ventricle to pulmonary conduits used to repair truncus arteriosus or pulmonary atresia with ventricular septal defect (VSD), or repaired malposition of the great arteries with VSD. This situation is often well tolerated, even in cases of severe pulmonary regurgitation, and delivery might be normal. ${ }^{8}$ The fear of ventricular or atrial arrhythmia can sometimes lead to pre-pregnancy pulmonary valve replacement, if the functional status before pregnancy is greatly impaired.

(3) Cyanotic patients with Ebstein anomaly, palliated pulmonary atresia with VSD, univentricular heart physiology, excluding Eisenmenger reaction. Vasodilatation induced by pregnancy usually increases cyanosis and can cause the mother's condition to deteriorate. In addition, hypoxaemia influences fetal growth and is linked to a high incidence of miscarriage when oxygen saturation is less than $85 \%$ or when haemoglobin is more than $18 \mathrm{~g} / \mathrm{dl}(11.2 \mathrm{mmol} / \mathrm{l})$. A moderate cyanosis increases the incidence of low birth weight and premature birth, as well as the risk of maternal thromboembolic events. ${ }^{9}$

(4) Moderate alteration of left ventricular function. Careful evaluation cannot always formally predict risk. Regular review should be undertaken during pregnancy, and early delivery should be considered if there is progressive deterioration in left ventricular function.

(5) Residual aortic coarctation or moderate left outflow tract obstruction. In case of residual coarctation a high level of upper limb systemic pressure should not induce inappropriate medical treatment for hypertension because of possible impairment of blood supply to the placenta. Patients with moderate left sided outflow tract obstruction benefit from rest in hospitals.

For all these patients obstetricians, anaesthetists, and cardiologists should closely cooperate, ideally on the same site. Maternal cardiac risks are mainly atrial arrhythmias (groups 1, 2, and 3), cardiac failure (groups 1, 3, and 4), increasing cyanosis (group 3), and thromboembolic events (groups 3 and 4).

\section{Management}

PRECONCEPTION OUTPATIENT CLINIC

It is essential to:

- inform the patient about the risks as far as one can anticipate them;

- assess the functional ability with echocardiography and exercise testing before pregnancy;

- carefully consider the potential risk to the fetus of maternal medication with agents such as angiotensin 
converting enzyme (ACE) inhibitors (risk of oligohydramnios, fetal and neonatal renal failure ${ }^{10}$ ) or amiodarone (risk of fetal hypothyroidism);

- encourage pregnancy early in life;

- inform the patient about the recurrent risks of congenital heart defects (as high as 6\% in tetralogy of Fallot ${ }^{11}$ );

- inform the patient about prenatal diagnosis, not only of heart defects but also about associated problems such as genetic issues;

- consider giving multivitamins to the patients three months before pregnancy and during the first three months after - these have been proven to lower the incidence of fetal heart defect in the general population ${ }^{12}$;

- detect risk factors for main obstetric complications (preeclampsia, early preterm delivery, fetal death in utero).

\section{DURING PREGNANCY}

Clinical assessment associated with regular comparative echocardiograms is wise for all patients, but follow up requires an individual approach for each patient, especially in the presence of impaired right ventricular function. Fetal heart scanning is traditionally offered at 18 weeks. Fetal growth, especially in cyanotic women, has to be carefully monitored.

Questions from obstetricians should be addressed. Can the patient have:

- tocolysis by $\beta$ stimulation or calcium channel inhibitors? Yes, but this may result in hypotension

- steroids (at high doses) for pulmonary maturation in case of premature labour to avoid fetal pulmonary distress? Yes, but they can induce volume expansion

- elective induced labour with oxytocic or prostaglandin? Yes, but there is a risk of bradycardia, hypertension, myocardial ischaemia, and vasospasm

- spinal anaesthesia? Yes, but since volume expanding solutions will be given, the risk of cardiac failure should be compared to the benefit of analgesia which will minimise haemodynamic changes during labour

- arterial pressure monitoring during labour (in case of sacrificed subclavian arteries)? Yes, but via an intravascular line, if necessary

- antibiotic prophylaxis? For normal vaginal delivery it is not recommended by the American Heart Association. ${ }^{13}$ Although well documented cases of endocarditis are uncommon, they can be very severe and we use prophylaxis (ampicillin) at the start of the labour and up to 48 hours

- planned breastfeeding? Yes, if the mother is not given drugs that are dangerous for the newborn

\section{TREATING COMPLICATIONS}

Most antiarrythmic drugs are well tolerated and can be given at relatively low risk to treat atrial arrhythmias. ${ }^{14}$ Amiodarone is an exception because of the risk of fetal hypothyroidism. Recent data suggest that $\beta$ blockers may cause intrauterine growth retardation if they are administered during the first trimester. In complex heart defects with "fragile ventricles", the use of potentially proarrhythmic drugs should be avoided. Direct current cardioversion to terminate maternal arrhythmias is well tolerated and effective.

There is no consensus about use of anticoagulants in cyanotic patients but low molecular weight heparin can certainly be a good alternative to oral anticoagulation. In patients with mechanical valves, the classical scheme is not ideal. Subcutaneous heparin during the first trimester, while avoiding teratogenicity associated with an oral anticoagulant, carries the risk of thrombosis. Switching from oral anticoagulant to subcutaneous heparin at the end of pregnancy can lead to severe haemorrhagic complications, if premature delivery occurs. The efficacy and safety of low molecular weight heparin has not yet been assessed for these patients.

\section{Conclusion}

Women who have survived congenital heart disease into adulthood often have a strong desire to become pregnant. Optimum care of these potentially complicated pregnancies can only be achieved by a combined approach by cardiologists and obstetricians in specialist centres with an understanding of the obstetric and cardiac complications that can arise.

Pediatric Cardiology and Adult Cardiology,

LAURENCE ISERIN

Necker Hospital,

149, Rue de Sèvres,

75015 Paris,

France

laurence.iserin@nck.ap-hop-paris.fr

1 Oakley CM. Pregnancy and congenital heart disease. Heart 1997;8:12-14

2 Siu S, Chitayat D, Webb G. Pregnancy in women with congenital heart

3 Zuber M, Gautschi N, Oechslin E, et al. Outcome of pregnancy in women Zuber M, Gautschi N, Oechslin E, et al. Outcome of
with congenital shunt lesions. Heart 1999;81:271-5.

de Swiet M. Maternal mortality: confidential enquiries into maternal deaths de Swiet M. Maternal mortality: confidential enquiries into matern
in the United Kingdom. Am $\mathcal{F}$ Obstet Gynecol 2000;182:760-6.

5 Rossiter JP, Morales AJ, Repke JT et al. A prospective longitudinal evaluation of pregnancy in the Marfan syndrome. Am F Obstet Gynecol 1995;173: 599-606.

6 Genoni M, Jenni R, Hoerstrup SP, et al. Pregnancy after atrial repair for transposition of the great arteries. Heart 1999;81:276-7.

7 Connolly HM, Grogan M, Warnes CA. Pregnancy among women with congenitally corrected transposition of the great arteries. $\mathcal{F}$ Am Coll Cardiol 1999;33:1692-5.

8 Neumayer U, Somerville J. Outcome of pregnancies in patients with complex pulmonary atresia. Heart 1997;78:6-21.

9 Presbitero P, Somerville J, Stone S, et al. Pregnancy in cyanotic congenital heart disease. Outcome of mother and fetus. Circulation. 1994;89:673-6.

10 Feldcamp M, Jones KL, Ornoy A, et al. Postmarketing surveillance for angiotensin-converting enzyme inhibitor use during pregnancy-United angiotensin-converting enzyme inhibitor use during pregn
States, Canada and Israel 1987-1995. fAMA 1997;277:193.

11 Burn J, Brennan P, Little J, et al. Recurrence risks in offspring of adults with major heart defects: results from first cohort of British collaborative study Lancet 1998;351:311-16.

12 Botto LD, Mulinare J, Erickson JD. Occurrence of congenital heart defects in relation to maternal multivitamin use. Am f Epidemiol 2000;151:878-84.

13 Dajani S, Taubert KA, Wilson W, et al. Prevention of bacterial endocarditis: recommendations by the American Heart Association. FAMA 1997;22: 1794-801.

14 Joglar JA, Page RL. Treatment of cardiac arrhythmias during pregnancy: safety considerations. Drug Saf 1999;20:85-94. 